\title{
HIV-positive patients in the intensive care unit: A retrospective audit
}

\author{
P Mkoko, ${ }^{1}$ MB ChB, MMed (Medicine), FCP (SA); R I Raine, ${ }^{1,2}$ MB ChB, MMed (Medicine), FCP (SA) \\ ${ }^{1}$ Department of Medicine, Groote Schuur Hospital and Faculty of Health Sciences, University of Cape Town, South Africa \\ ${ }^{2}$ Department of Critical Care, Groote Schuur Hospital and Faculty of Health Sciences, University of Cape Town, South Africa
}

Corresponding author: P Mkoko(mkoko25@me.com)

\begin{abstract}
Background. The indications for and outcomes of intensive care unit (ICU) admission of HIV-positive patients in resource-poor settings such as sub-Saharan Africa are unknown.

Objective. To identify indications for ICU admission and determine factors associated with high ICU and hospital mortality in HIV-positive patients.

Methods. We reviewed case records of HIV-positive patients admitted to the medical and surgical ICUs at Groote Schuur Hospital, Cape Town, South Africa, from 1 January 2012 to 31 December 2012.

Results. Seventy-seven HIV-positive patients were admitted to an ICU, of whom two were aged $<18$ years and were excluded from the final analysis. HIV infection was newly diagnosed in $37.3 \%$ of the patients admitted during the study period. HIV-positive patients had a median CD4 count of 232.5 (interquartile range 59 - 459) cells/ $\mu \mathrm{L}$. Respiratory illness, mainly community-acquired pneumonia, accounted for $30.7 \%$ of ICU admissions. ICU and hospital mortality rates were $25.3 \%$ and $34.7 \%$, respectively. Predictors of ICU mortality included an Acute Physiology and Chronic Health Evaluation II (APACHE II) score $>13$ (odds ratio (OR) 1.4, 95\% confidence interval (CI) 1.1 - 1.7; $p=0.015)$, receipt of renal replacement therapy (RRT) (OR 2.2, 95\% CI $1.2-4.1 ; p=0.018)$ and receipt of inotropes (OR 2.3, $95 \%$ CI 1.6 - 3.4; $p<0.001$ ). Predictors of hospital mortality were severe sepsis on admission (OR 2.8, 95\% CI $0.9-9.1 ; p=0.07$ ), receipt of RRT (OR 1.9, $95 \%$ CI $1.0-3.6 ; p=0.056$ ) and receipt of inotropic support (OR 2.0, 95\% CI $1.4-3.2 ; p<0.001$ ). Use of highly active antiretroviral therapy (HAART), CD4 count, detectable HIV viral load and diagnosis at ICU admission did not predict ICU or hospital mortality.

Conclusions. Respiratory illnesses remain the main indication for ICU in HIV-positive patients. HIV infection is often diagnosed late, with patients presenting with life-threatening illnesses. Severity of illness as indicated by a high APACHE II score, multiple organ dysfunction requiring inotropic support and RRT, rather than receipt of HAART, CD4 count and diagnosis at ICU admission, are predictors of ICU and hospital mortality.
\end{abstract}

S Afr Med J 2017;107(10):877-881. DOI:10.7196/SAMJ.2017.v107i10.12298

HIV/AIDS is a major public health burden, with an estimated 35.3 million people infected globally. ${ }^{[1]}$ Sub-Saharan Africa (SSA) bears the brunt of the pandemic, with 25 million HIV-positive people living in the region. ${ }^{[1]}$ The advent of highly active antiretroviral therapy (HAART) has changed the natural history of HIV/AIDS, with a reported decline in mortality from 24.5 per 100 person-years in 1995 to 8.9 per 100 in $1997,{ }^{[2]}$ and reportedly 2.1 per 100 in $2010 .{ }^{[3]}$ Today HIV-positive patients in high-income countries are reported to have a life expectancy approaching that of the general population. ${ }^{[4]}$ Despite evidence of the role of HAART in decreasing morbidity and mortality in HIV-positive patients, in resource-poor settings many people living with HIV do not have access to HAART or receive treatment late. Globally, only 9.7 million people were estimated to be receiving HAART in 2012. ${ }^{[5]}$ Many HIV-positive people present with serious illnesses that may require intensive care unit (ICU) admission, ${ }^{[6]}$ and a large proportion of these are unaware of their HIV status. ${ }^{[7]}$ Data from high-income countries suggest that $7-12 \%$ of HIV-positive patients admitted to hospital are treated in the ICU. ${ }^{[8,9]}$

Historically, respiratory failure accounted for the majority of ICU admissions in the HIV-positive patient population, with an associated mortality of $\sim 70 \% .{ }^{[9,10]}$ However, contemporary evidence from high-income countries suggests that ICU outcomes are similar to those of HIV-negative patients and that the indications for ICU are mostly not related to opportunistic infections (OIs). ${ }^{[10,11]}$
ICU utilisation and outcomes for HIV-positive patients in poorly resourced settings, where access to both intensive care and HAART is limited, have not been widely studied. With an increasing prevalence of HIV and an unchanging (and at times decreasing) number of available ICU beds, it is imperative to know the profile and outcome of HIV-positive patients requiring ICU admission in a resource-poor setting such as South Africa (SA).

\section{Objective}

To identify indications for ICU admission and determine factors associated with high ICU and hospital mortality in HIV-positive patients admitted to ICUs in a resource-limited setting. This will help in determining the appropriateness of offering ICU care to HIVpositive patients and in the appropriate development of policies and planning for allocation of limited resources.

\section{Methods}

\section{Study design and patient population}

The study was a retrospective folder review conducted from 1 January 2012 to 31 December 2012 in the medical and surgical ICUs at Groote Schuur Hospital (GSH), Cape Town, SA. GSH is a tertiary hospital affiliated to the University of Cape Town (UCT), and is an 867-bed institution with 16 general medicine and general surgery adult ICU beds (excluding the coronary care unit, the neurosurgical ICU and 
the cardiothoracic ICUs). The GSH ICUs admit all patients deemed to require ICU care, irrespective of the need for ventilation. At the time of the study, GSH did not have a high-care unit. The GSH ICUs have no ICU admission criteria, admission being at the discretion of the treating or referring physician and the ICU physician on call at the time of ICU referral.

Patients were enrolled in the study if they were aged $>18$ years at the time of ICU admission, known to be HIV-positive, or newly diagnosed with HIV in the index admission.

Ward admission books and CLINICOM (a health information system including demographic and clinical patient data and providing a single electronic patient record that is accessible throughout the Western Cape Province of SA) records were interrogated for information on all the patients admitted to an ICU during the study period. Patient folders and laboratory records were examined for the results of HIV tests done during and prior to the index admission and for patient diagnoses during ICU admission.

\section{Ethical approval}

The study was carried out with the approval of the GSH and UCT Human Subjects Research Ethics Committee (ref. no. 044/2013). The need to obtain informed consent was waived, as this was a retrospective study and no identifying details would be included.

\section{Case definition}

Patients were coded as HIV-negative if they had had a negative HIV test 3 months prior to the index admission, and as untested if there was no documented HIV test result in the folder or no recorded HIV test in the National Health Laboratory Service electronic records. HIV infection was diagnosed by two positive antibody tests or one positive antibody test and a confirmatory Western blot test. No HIV testing was offered or done for the purpose of this study.

\section{Data collection}

A standardised form was used to collect demographic information, including age, sex and ethnic group. Details of length of ICU and hospital stay, use of mechanical ventilation and inotropic support were also recorded. The most recent CD4 count and HIV viral load, when available, were recorded. The Acute Physiology and Chronic Health Evaluation II (APACHE II) score was calculated using laboratory values obtained within 24 hours of ICU admission. Use of HAART before hospital admission was recorded. The presence of acute kidney injury (AKI) (as defined by Kidney Disease Improving Global Outcomes) on admission to the ICU and the use of renal replacement therapy (RRT) were documented. ${ }^{[12]}$ Diagnoses at ICU admission were categorised by organ system and severity of sepsis. ${ }^{[13]}$

\section{Statistical analysis}

Data analysis was performed using SPSS version 20 (IBM, USA). Normality of data was tested using the Kolmogorov-Smirnov test. Normally distributed data are presented as means (standard deviation $(\mathrm{SD})$ ) or, where highly skewed, as medians (interquartile range (IQR)); discrete data are presented as numbers (percentages). The $\chi^{2}$ test or Fisher's exact test was utilised to compare discrete data, as appropriate. In an attempt to identify factors associated with ICU and hospital mortality in HIV-positive individuals, HIV-positive patients who died in the ICU were compared with HIV-positive patients who were discharged alive from the ICU, and HIV-positive patients who died in hospital were compared with HIV-positive patients who were discharged alive. Analysis of variance with post-hoc Bonferroni correction was used to explore whether there were differences between the groups. Bivariate correlations were assessed using the Pearson $r$ and Spearman $r_{S}$ coefficients, as appropriate. All statistical tests were two-tailed, with $p<0.05$ considered significant.

\section{Results}

From 1 January 2012 to 31 December 2012, 806 patients were admitted to the ICUs. Of these, 77 were HIV-positive, giving an incidence of 9.6 per 100 person-years. Two patients were aged $<18$ years and were excluded from the final analysis (Fig. 1). Two hundred and thirty-seven patients (29.4\%) were HIV-uninfected or had tested HIV-negative during the 3 months prior to ICU admission. HIV status was unknown for 492 patients admitted to the ICUs (61.0\%).

\section{Patient demographic and clinical characteristics at ICU admission}

A total of 75 patients were included in the analysis, with a mean (SD) age of 36.5 (9.6) years (Table 1). In keeping with the epidemiology of HIV infection in SSA, the majority of patients were female (64.0\%). The CD4 count was available for 74 of the 75 patients. The median CD4 count was 232.5 (IQR 59 - 459) cells/ $\mu$ L. The HIV viral load was available for 27 patients, all of whom were receiving HAART at the time of ICU admission. Thirteen of the 27 patients had a detectable HIV viral load. The mean HIV viral load was 2218556.5 RNA copies/mL and the median HIV viral load was 20146.5 (IQR 372.75 25 898.75) RNA copies/mL. More than a third of the patients (37.3\%) were newly diagnosed with HIV during the index hospital admission (Table 1).

Thirty-nine patients $(52.0 \%)$ were not receiving HAART. The mean (SD) duration of HAART was 6.3 (17.5) months. No patients were started on antiretroviral therapy during their ICU stay.

ICU admission occurred after a median of 2 (IQR 1 - 6) days in the referring unit; 33 patients (44.0\%) were admitted to the ICU within 24 hours of arrival in hospital, 17 (22.7\%) between 24 and 72 hours after arrival, and $25(33.3 \%)>72$ hours after arrival (Fig. 2). Thirtyone patients $(41.3 \%)$ were referred to the ICU from the general medical wards, $20.0 \%$ from casualty (medical emergency), $16.0 \%$ from the general surgical wards, $12.0 \%$ from the trauma ward and $10.7 \%$ from maternity (Table 1). Approximately one-third (30.7\%) of the patients were admitted to the ICU for respiratory illnesses. Fifteen of the patients with respiratory illness had communityacquired pneumonia, four had pulmonary tuberculosis (initially referred to the ICU as community-acquired pneumonia) and the other four had Pneumocystis jirovecii pneumonia. The diagnosis of P. jirovecii pneumonia was made after ICU admission. Two of the

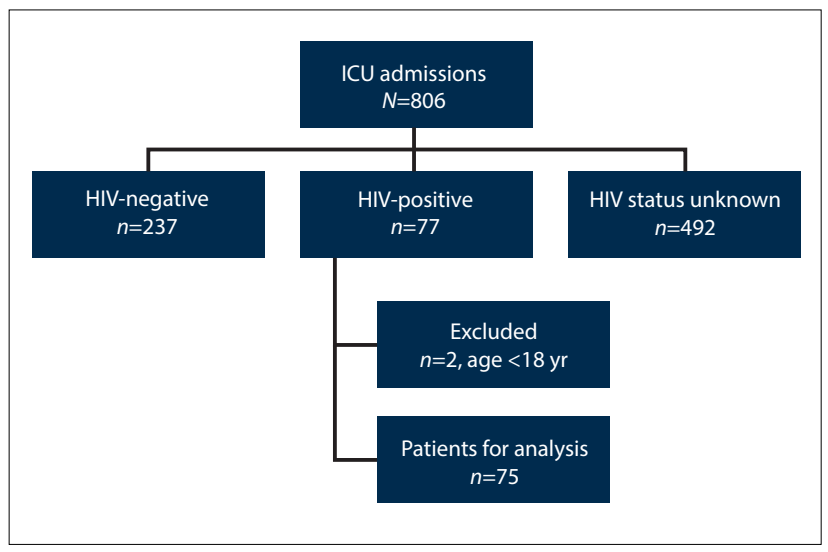

Fig. 1. Flow diagram showing patient enrolment. $(I C U=$ intensive care unit. $)$ 


\begin{tabular}{|c|c|}
\hline Age (years), mean (SD) & $36.5(9.6)$ \\
\hline Female $(\%)$ & 64.0 \\
\hline \multicolumn{2}{|l|}{ Race (\%) } \\
\hline Black & 84.0 \\
\hline Mixed race & 16.0 \\
\hline Newly diagnosed HIV (\%) & 37.3 \\
\hline On HAART (\%) & 48.0 \\
\hline Duration of HAART (months), range & $0-72$ \\
\hline CD4 count (cells/ $\mu \mathrm{L})$, median (IQR) & $232.5(59-459)$ \\
\hline Detectable HIV viral load (\%) & 18.7 \\
\hline \multicolumn{2}{|l|}{ Prior HIV-related illnesses (\%) } \\
\hline Tuberculosis & 25.3 \\
\hline Syphilis & 1.3 \\
\hline HIV-associated nephropathy & 1.3 \\
\hline \multicolumn{2}{|l|}{ Source of referral (\%) } \\
\hline Emergency unit* & 32.0 \\
\hline Medical ward & 41.3 \\
\hline Surgical ward & 16.0 \\
\hline Maternity & 10.7 \\
\hline $\begin{array}{l}\text { Number of days in the referring unit, median } \\
\text { (IQR) }\end{array}$ & $2(1-45)$ \\
\hline \multicolumn{2}{|l|}{ Diagnoses at ICU admission (\%) } \\
\hline Respiratory illness & 30.7 \\
\hline Severe sepsis & 13.3 \\
\hline Trauma & 12.0 \\
\hline Drug toxicity and poisoning & 8.0 \\
\hline Neurological illness & 9.3 \\
\hline Elective surgery & 8.0 \\
\hline Other $^{\dagger}$ & 18.7 \\
\hline APACHE II score, mean (SD) & $21.6(8.4)$ \\
\hline AKI on admission (\%) & 26.7 \\
\hline Mechanical ventilation (\%) & 90.7 \\
\hline RRT (\%) & 32.0 \\
\hline Inotropic support (\%) & 50.7 \\
\hline \multicolumn{2}{|l|}{ ICU complications (\%) } \\
\hline AKI & 16.0 \\
\hline Sepsis & 25.3 \\
\hline AKI and sepsis & 17.3 \\
\hline Number of days in ICU, median (IQR) & $4(2-8)$ \\
\hline Number of days in hospital, median (IQR) & $16(9-34)$ \\
\hline \multicolumn{2}{|c|}{$\begin{array}{l}\mathrm{SD}=\text { standard deviation; HAART = highly active antiretroviral therapy; IQR = } \\
\text { interquartile range; ICU = intensive care unit; APACHE II = Acute Pyysiology and } \\
\text { Chronic Health Evaluation II; AKI = acute kidney injury; RRT = renal replacement } \\
\text { therapy } \\
\text { *IIncludes medical emergency (casualty) and trauma unit. } \\
\text { 'Includes patients admitted with renal illness, malaria, gastrointestinal illness, emergency } \\
\text { surgery and cardiac illness. }\end{array}$} \\
\hline
\end{tabular}

patients diagnosed with $P$. jirovecii pneumonia had a new diagnosis of HIV infection. The other two patients with $P$. jirovecii pneumonia were known to be HIV-positive and were receiving HAART; their CD 4 counts were $<150$ cells $/ \mu \mathrm{L}$, and both had a detectable HIV viral load. Ten patients had pulmonary tuberculosis and were receiving antituberculosis therapy before ICU admission. Nineteen patients had previous tuberculosis, which had been fully treated.

\section{Patient clinical characteristics during the ICU stay}

The majority of the patients (90.7\%) required mechanical ventilation, and $50.7 \%$ required inotropic support. Fifty-five patients (73.3\%) had AKI on admission to the ICU. RRT was offered to 24 patients

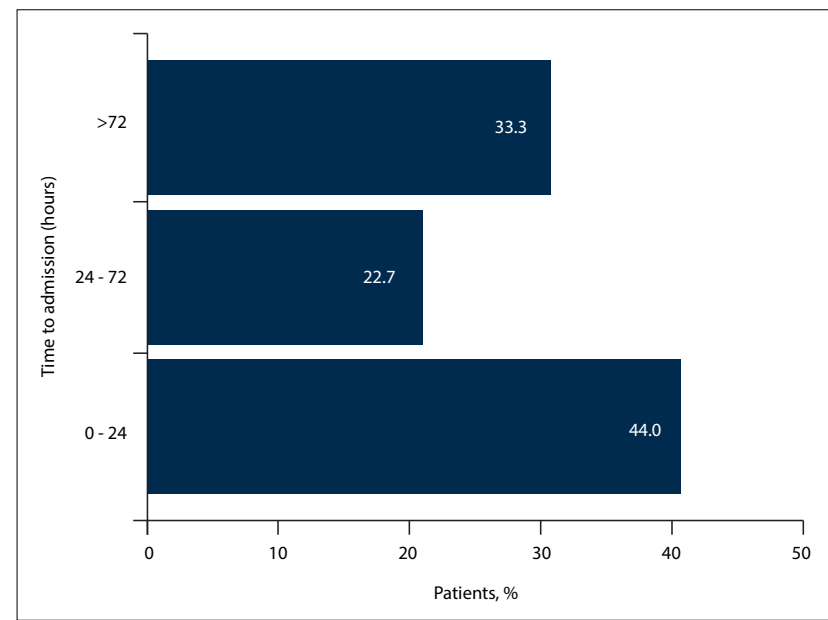

Fig. 2. Time in the referring unit prior to intensive care unit admission.

(32.0\%). Patients presented with a mean (SD) APACHE II score of 21.6 (8.4). The median numbers of days in the ICU and in hospital were 4 (IQR 2 - 8) and 16 (IQR 9 - 34), respectively.

\section{ICU and hospital mortality}

Nineteen patients (25.3\%) died in the ICU, an additional 7 patients died in hospital after ICU discharge (9.3\%), and 49 (65.3\%) were discharged from hospital alive. Use of RRT was significantly associated with mortality in the ICU (odds ratio (OR) 2.2, 95\% confidence interval (CI) $1.2-4.1 ; p=0.018)$, but not with hospital mortality (OR 1.9, 95\% CI $1.0-3.6 ; p=0.056$ ) (Table 2). The use of inotropes in the ICU was associated with both ICU (OR 2.3, 95\% CI $1.6-3.4 ; p<0.001$ ) and hospital mortality (OR 2.0, 95\% CI 1.4 $3.2 ; p<0.001)$. An APACHE II score of $>13$ at ICU admission was also significantly associated with increased ICU mortality (OR 1.4, 95\% CI $1.1-1.7 ; p=0.015$ ), but not hospital mortality (OR 1.3, 95\% CI 1.0 - 1.6; $p=0.066)$. The number of days in the referring unit prior to ICU admission did not predict death in the ICU $(p=0.075)$ or in hospital ( $p=0.191$ ). Factors including CD4 count, use of HAART, mechanical ventilation, serum albumin and whether the patient was known to have or was newly diagnosed with HIV infection were not significantly associated with increased ICU or hospital mortality.

\section{Discussion}

The key findings of our study were: (i) that respiratory illnesses remain the major indication for ICU admission in HIV-positive patients; (ii) that there were ICU and hospital mortality rates of $25.3 \%$ and $34.7 \%$, respectively, in a contemporary SA setting; and (iii) that factors associated with poor short-term outcomes in HIV-positive patients admitted to the ICU were the use of RRT, vasopressor support and an APACHE II score $>13$. To our knowledge, this is the first study that has determined the outcomes of HIV-positive adults admitted to an SA ICU. These results have implications for resource allocation and management of HIV-positive patients requiring ICU care in resource-constrained settings. They suggest that sicker patients, as suggested by higher APACHE II scores and the need for organ support such as RRT and inotropes in the setting of HIV infection, have worse outcomes. In resource-limited settings, ICU care may have to be withheld from HIV-positive patients who meet the above criteria. Furthermore, these results support the general criteria for ICU admission used in the SA public sector, which take into account the severity of the clinical presentation and the likelihood of reversibility of organ dysfunction. ${ }^{[14]}$ 
Table 2. Patient outcomes according to specific variables

\begin{tabular}{|c|c|c|c|c|c|c|}
\hline & $\begin{array}{l}\text { Death in } \\
\text { ICU, } n\end{array}$ & OR $(95 \% \mathrm{CI})$ & $p$-value & $\begin{array}{l}\text { Death in } \\
\text { hospital, } n\end{array}$ & OR $(95 \% \mathrm{CI})$ & $p$-value \\
\hline Female $(N=48)$ & 15 & $1.2(0.8-1.7)$ & 0.403 & 16 & $0.9(0.7-1.4)$ & 0.746 \\
\hline Respiratory illnesses $(N=23)$ & 6 & $1.1(0.5-2.3)$ & 0.755 & 7 & $1.8(0.4-1.7)$ & 0.609 \\
\hline Severe sepsis $(N=10)$ & 3 & $1.1(0.3-3.9)$ & 0.880 & 6 & $2.8(0.9-9.1)$ & 0.071 \\
\hline AKI on admission $(N=20)$ & 7 & $1.7(0.8-3.6)$ & 0.163 & 8 & $1.3(0.6-2.7)$ & 0.558 \\
\hline Mechanical ventilation $(N=68)$ & 20 & $1.0(0.9-1.2)$ & 0.396 & 25 & $1.1(1.0-1.2)$ & 0.234 \\
\hline Renal replacement therapy $(N=24)$ & 11 & $2.2(1.2-4.1)$ & 0.018 & 12 & $1.9(1.0-3.6)$ & 0.056 \\
\hline Inotropic support $(N=38)$ & 18 & $2.3(1.6-3.4)$ & $<0.001$ & 20 & $2.0(1.4-3.2)$ & 0.001 \\
\hline APACHE II >13 $(N=57)$ & 20 & $1.4(1.1-1.7)$ & 0.015 & 23 & $1.3(1.0-1.6)$ & 0.066 \\
\hline Newly diagnosed HIV $(N=28)$ & 10 & $1.4(0.8-2.6)$ & 0.251 & 11 & $1.2(0.7-2.2)$ & 0.516 \\
\hline Not on HAART $(N=39)$ & 13 & $1.3(0.8-2.0)$ & 0.284 & 16 & $1.3(0.9-2.0)$ & 0.228 \\
\hline Detectable HIV viral load $(N=13)$ & 4 & $1.3(0.6-2.8)$ & 0.580 & 5 & $1.3(0.6-2.7)$ & 0.586 \\
\hline $\mathrm{CD}_{4}$ count $<200$ cells $/ \mu \mathrm{L}(N=35)$ & 12 & $1.4(0.9-2.3)$ & 0.183 & 15 & $1.5(0.9-2.3)$ & 0.118 \\
\hline Time in the referring unit (hours) & & & 0.075 & & & 0.191 \\
\hline$<24(N=33)$ & 9 & & & 12 & & \\
\hline $24-72(N=17)$ & 1 & & & 3 & & \\
\hline$>72(N=25)$ & 9 & & & 14 & & \\
\hline
\end{tabular}

Intensive care for HIV-positive patients was initially remarkable for high ICU and hospital mortality, ${ }^{[15,16]}$ and in keeping with previous reports we found an ICU mortality rate of $25.3 \%{ }^{[10,17,18]}$ Respiratory illnesses remain the leading diagnoses at ICU admission in HIVpositive patients. ${ }^{[9,10]}$ It has been reported that mechanical ventilation for respiratory illnesses is associated with poor ICU outcomes. ${ }^{[9,19-22]}$ In this SA cohort, respiratory illnesses represented the most common indication for ICU admission, but were not associated with increased ICU and hospital mortality. We also did not find an association of mechanical ventilation with ICU and hospital mortality. In a study by Casalino et al.. ${ }^{[22]} 45 \%$ of the patients with respiratory illnesses had $P$. jirovecii pneumonia. In the HAART era, the incidence of $P$. jirovecii pneumonia in HIV-positive patients admitted to ICUs has decreased to $3-9 \% \cdot{ }^{[10,11]} \mathrm{Up}$ to $50 \%$ of HIV-positive patients are unaware of their HIV status prior to ICU admission. ${ }^{[7,23-25]}$ Compared with patients who are known to be HIV-positive prior to ICU admission, this patient group is characterised by pronounced immunosuppression and typically admitted with AIDS-related diagnoses such as $P$. jirovecii pneumonia. ${ }^{[10,23,25-27]}$ The frequency of newly diagnosed HIV-positive patients at ICU admission has remained relatively the same in the HAART era as in the pre-HAART era, but the frequency of OIs in the ICU has decreased. ${ }^{[7,9]}$ Only $5.3 \%$ of our patients had $P$. jirovecii pneumonia. The diagnoses of $P$. jirovecii pneumonia was made during their ICU stay. $P$. jirovecii pneumonia, the need for mechanical ventilation in $P$. jirovecii pneumonia, development of a pneumothorax in $P$. jirovecii pneumonia and ICU admission for an AIDS-related diagnosis are widely thought to be related to high ICU and hospital mortality. ${ }^{[8,17,19,24,28]}$ We do not have information on the number of HIV-positive patients who might have qualified for ICU care but were not referred to an ICU or were turned down for admission. This would help determine the reasons behind the denial of ICU care in this patient population and whether physician attitudes towards ICU care for HIV-positive patients have changed.

The severity of the acute event as marked by high APACHE II scores, ${ }^{[9.28-30]}$ the use of inotropes ${ }^{[27,31,32]}$ and $\mathrm{RRT}^{[33]}$ is reported to be associated with poor short-term outcomes. In this study, we found that a high APACHE II score, use of inotropes and RRT were significantly associated with both ICU and hospital mortality in HIV-positive patients. Their presence in areas where resources are constrained could be used to determine whether ICU admission is warranted or intensive support should be initiated.

Between $24 \%$ and $50 \%$ of HIV-positive patients receive HAART prior to ICU admission. ${ }^{[8,10,23,26]}$ Receipt of HAART prior to ICU admission has been associated with contradictory results in terms of association with short-term outcomes. Some authors have reported increased survival rates in patients on HAART prior to ICU admission, while others have reported that receipt of HAART did not predict short-term outcomes. ${ }^{[7,8,11,27]}$ In our study, $48.0 \%$ of the patients were on HAART prior to ICU admission, but HAART prior to ICU admission did not predict ICU and hospital survival. Reports have shown that HIV viral load and CD4 count did not predict ICU and hospital survival. ${ }^{[1,27,28]}$ Similarly, in our study we did not find any statistically significant association between detectable HIV viral load, low CD4 count and short-term outcomes in terms of either ICU or hospital mortality.

Up to $50 \%$ of HIV-positive persons have severe sepsis at ICU admission, or suffer from severe sepsis during their ICU stay. ${ }^{[32]}$ Many infections responsible for sepsis in this patient population are nosocomial, followed by AIDS-related infections. ${ }^{[29]}$ Ward stay prior to ICU admission is a risk factor for sepsis in HIV-positive patients. ${ }^{[32]}$ Severe sepsis has been reported to be associated with poor ICU outcomes in HIV-positive patients. ${ }^{[32]}$ In our study, 13.3\% of the patients had a diagnosis of severe sepsis at ICU admission, and $42.6 \%$ acquired severe sepsis during their ICU stay. We did not find an association between severe sepsis and death in this cohort.

The fact that $52.0 \%$ of the patients in our cohort were not receiving HAART, and $18.7 \%$ had a detectable HIV viral load while receiving HAART, suggests that improving ICU care plays a major role in the better outcomes of HIV-positive patients in the ICU. In our hospital, we have adopted lung-protective ventilation, surviving sepsis guidelines and appropriate glucose control in our ICUs. ${ }^{[34,35]}$ There were very few case of $P$. jirovecii pneumonia, which may account for the apparent lack of association between respiratory illnesses, mechanical ventilation and death.

\section{Study limitations}

Our study has several important limitations: $(i)$ it is a singlecentre study; (ii) it suffers from the limitations and biases of its 
retrospective nature; (iii) we were not able to find and document the microbiological causes of all the cases of community-acquired pneumonia, which could offer an opportunity for prophylaxis; and (iv) most importantly, $61.0 \%$ of our ICU population did not have an HIV test. Considering that our community has a high prevalence of HIV infection, this represents a missed opportunity for testing and potential treatment.

\section{Conclusions}

This study shows that critical care outcomes for HIV-positive patients at GSH are comparable to those in the developed world. As these are findings from a tertiary care unit, they are not representative of the rest of the country. The study highlights the fact that ICU outcomes for this patient population depend on the severity of the acute illness, irrespective of receipt of HAART and immune status. More research is needed that is representative of poorer provinces of SA and the rest of the African continent.

Acknowledgements. We thank Profs Ntobeko Ntusi, Ikechi Okpechi and Sipho Dlamini and Dr Richard Raine for their constant support. Prof. Ntobeko Ntusi performed the statistical analysis.

Author contributions. PM designed the study, performed the clinical research and wrote the article, and RIR supervised the project.

Funding. None.

Conflicts of interest. None.

1. Joint United Nations Programme on HIV/AIDS. UNAIDS Report on the Global AIDS Epidemic 2013 Geneva: UNAIDS, 2013. http://www.unaids.org/en/resources/documents/2013/20130923_UNAIDS_ Global_Report_2013 (accessed 20 April 2015).

2. Palella FJ Jr, Delaney KM, Moorman AC, et al., HIV Outpatient Study Investigators. Declining morbidity and mortality among patients with advanced human immunodeficiency virus infection. N Engl J Med 1998;338(13):853-860. https://doi.org/10.1056/NEJM199803263381301

3. Moore RD, Keruly JC, Bartlett JG. Improvement in the health of HIV-infected persons in care: Reducing disparities. Clin Infect Dis 2012;55(9):1242-1251. https://doi.org/10.1093/cid/cis654

4. Sami H, Cescon A, Hogg RS, et al. Closing the gap: Increases in life expectancy among treated Samji H, Cescon A, Hogg RS, et al. Closing the gap: Increases in life expectancy among treated
HIV-positive individuals in the United States and Canada. PLoS One 2013;8(12):e81355. https://doi. HIV-positive individuals in the Un
org/10.1371/journal.pone.0081355

5. World Health Organization/Joint United Nations Programme on HIV/AIDS/ United Nations Children's Fund. Global Update on HIV Treatment 2013: Results, Impact and Opportunities. 2013. Geneva: WHO/UNAIDS/UNICEF, 2013. http://apps.who.int/iris/bitstream/10665/85326/1/9789241505734_ eng.pdf (accessed 20 April 2015).

6. Shrosbree J, Campbell LJ, Ibrahim F, et al. Late HIV diagnosis is a major risk factor for intensive care unit admission in HIV-positive patients: A single centre observational cohort study. BMC Infect Dis 2013;13(1):23. https://doi.org/10.1186/1471-2334-13-23

7. Casalino E, Wolff M, Ravaud P, et al. Impact of HAART advent on admission patterns and survival in HIV-infected patients admitted to an intensive care unit. AIDS 2004;18(10):1429-1433. https://doi. org/10.1097/01.aids.0000131301.55204.a7

8. Khouli H, Afrasiabi A, Shibli M, et al. Outcome of critically ill human immunodeficiency virusinfected patients in the era of highly active antiretroviral therapy. J Intensive Care Med 2005;20(6):327333. https://doi.org/10.1177/0885066605281087

9. Afessa B, Green B. Clinical course, prognostic factors, and outcome prediction for HIV patients in 9. Afessa B, Green B. Clinical course, prognostic factors, and outcome prediction for HIV patients in
the ICU: The PIP (Pulmonary Complications, ICU Support, and Prognostic Factors in Hospitalized the ICU: The PIP (Pulmonary Complications, ICU Support, and Prognostic Factors in Hosp
Patients with HIV) study. Chest 2000;118(1):138-145. https://doi.org/10.1378/chest.118.1.138

10. Powell K, Davis JL, Morris AM, et al. Survival for patients with HIV admitted to the ICU continues to improve in the current era of combination antiretroviral therapy. Chest 2009;135(1):11-17. https:// doi.org/10.1378/chest.08-0980

11. Narasimhan M, Posner AJ, DePalo VA, et al. Intensive care in patients with HIV infection in the era of highly active antiretroviral therapy. Chest 2004;125(5):1800-1804. https://doi.org/10.1378/ chest. 125.5 .1800
12. Khwaja A. KDIGO clinical practice guidelines for acute kidney injury. Nephron Clin Pract 2012;120(4):c179-c184. https://doi.org/10.1159/000339789

13. Levy MM, Fink MP, Marshall JC, et al. 2001 SCCM/ESICM/ACCP/ATS/SIS International Sepsis Definitions Conference. Crit Care Med 2003;31(4):1250-1256. https://doi.org/10.1007/s00134-003$1662-\mathrm{x}$

14. Naidoo KS, Singh JA, Lalloo UG. HIV-AIDS and admission to intensive care units - a comparison of India, Brazil and South Africa. S Afr J HIV Med 2013;14(1):15-16. https://doi.org/10.4102/sajhivmed. v14i1.96

15. Wachter RM, Russi MB, Bloch DA, et al. Pneumocystis carinii pneumonia and respiratory failure in AIDS: Improved outcomes and increased use of intensive care units. Am Rev Respir Dis 1991;143(2):251-256. https://doi.org/10.1164/ajrccm/143.2.251

16. Hawley PH, Ronco JJ, Guillemi SA, et al. Decreasing frequency but worsening mortality o acute respiratory failure secondary to AIDS-related Pneumocystis carinii pneumonia. Chest 1994;106(5):1456-1459. http://dx.doi.org/10.1378/chest.106.5.1456

17. Adlakha A, Pavlou M, Walker DA, et al. Survival of HIV-infected patients admitted to the intensive care unit in the era of highly active antiretroviral therapy. Int J STD AIDS 2011;22(9):498-504. https:/ doi.org/10.1258/ijsa.2011.010496

18. Yoon C, Weir D, Greene M, Jarlsberg LG, Davis JL, Huang L. Have we plateaued? Outcomes of HIVinfected patients admitted to the intensive care unit in the combined antiretroviral therapy era. D105. Lung Complications of Chronic HIV Infection: Prevalence, Pathogenesis, and Physiology. D105. Lung Complications of Chronic HIV Infection: Prevalence, Pathogenesis, and Physiology.
Am J Resp Crit Care Med 2011;183:A6271. http://www.atsjournals.org/doi/abs/10.1164/airccmAm J Resp Crit Care Med 2011;183:A6271. http://www.atsjournals.org/do/
conference.2011.183.1_MeetingAbstracts.A6271 (accessed 5 September 2017).

19. Gill JK, Greene L, Miller R, et al. ICU admission in patients infected with the human immunodeficiency viluK, Greene L, Miller R, et al. ICU admission in patients infected with the human immunodeficiency
virus - a multicentre survey. Anaesthesia 1999;54(8):727-732. https://doi.org/10.1046/j.1365-2044. virus - a mulfice
$1999.00903 . x$

20. Rosen MJ, Clayton K, Schneider RF, et al., Pulmonary Complications of HIV Infection Study Group Intensive care of patients with HIV infection: Utilization, critical illnesses, and outcomes. Am J Respi Crit Care Med 1997;155(1):67-71. https://doi.org/10.1164/ajrccm.155.1.9001291

21. De Palo VA, Millstein BH, Mayo PH, et al. Outcome of intensive care in patients with HIV infection. Chest 1995;107(2):506-510. https://doi.org/10.1378/chest.107.2.506

22. Casalino E, Mendoza-Sassi G, Wolff M, et al. Predictors of short- and long-term survival in HIVinfected patients admitted to the ICU. Chest 1998;113(2):421-429. http://dx.doi.org/10.1378/ chest.113.2.421

23. Palacios R, Hidalgo A, Reina C, et al. Effect of antiretroviral therapy on admissions of HIV-infected patients to an intensive care unit. HIV Med 2006;7(3):193-196. https://doi.org/10.1111/j.1468-
$1293.2006 .00353 . \mathrm{x}$

24. Miller RF, Allen E, Copas A, et al. Improved survival for HIV infected patients with severe Pneumocystis jirovecii pneumonia is independent of highly active antiretroviral therapy. Thorax 2006;61(8):716-721. https://doi.org/10.1136/thx.2005.055905

25. Croda J, Croda MG, Neves A, et al. Benefit of antiretroviral therapy on survival of human immunodeficiency virus-infected patients admitted to an intensive care unit. Crit Care Med 2009;37(5):1605-1611. https://doi.org/10.1097/CCM.0b013e31819da8c

26. Dickson SJ, Batson S, Copas AJ, et al. Survival of HIV-infected patients in the intensive care unit in the era of highly active antiretroviral therapy. Thorax 2007;62(11):964-968. https://doi.org/10.1136/ thx.2006.072256

27. Barbier F, Coquet I, Legriel S, et al. Etiologies and outcome of acute respiratory failure in HIV-infected patients. Intensive Care Med 2009;35(10):1678-1686. https://doi.org/10.1007/s00134-009-1559-4

28. Morris A, Creasman J, Turner J, et al. Intensive care of human immunodeficiency virus-infected patients during the era of highly active antiretroviral therapy. Am J Respir Crit Care Med 2002;166(3):262-267. https://doi.org/10.1164/rccm.2111025

29. Greenberg JA, Lennox JL, Martin GS, et al. Outcomes for critically ill patients with HIV and severe sepsis in the era of highly active antiretroviral therapy. J Crit Care 2012;27(1):51-57. https://doi. sepsis in the era of highly active
org $/ 10.1016 /$ j.jcrc.2011.08.015

30. Amancio FF, Lambertucci IR, Cota GF, et al. Predictors of the short- and long-term survival of HIVinfected patients admitted to a Brazilian intensive care unit. Int J STD AIDS 2012;23(10):692-697. infected patients admitted to a Brazilian
https://doi.org/10.1258/ijsa.2012.011389

31. Morquin D, le Moing V, Mura T, et al. Short- and long-term outcomes of HIV-infected patients admitted to the intensive care unit: Impact of antiretroviral therapy and immunovirological status. Ann Intensive Care 2012;2(1):25. https://doi.org/10.1186/2110-5820-2-25

32. Japiassu AM, Amancio RT, Mesquita EC, et al. Sepsis is a major determinant of outcome in critically il HIV/AIDS patients. Crit Care 2010;14(4):R152. https://doi.org/10.1186/cc9221

33. Arendse C, Okpechi I, Swanepoel C. Acute dialysis in HIV-positive patients in Cape Town, South Africa. Nephrology (Carlton) 2011;16(1):39-44. https://doi.org/10.1111/j.1440-1797.2010.01358.x

34. Dellinger RP, Levy MM, Rhodes A, et al. Surviving sepsis campaign: International guidelines for management of severe sepsis and septic shock: 2012. Crit Care Med 2013;41(2):580-637. https://doi. org/10.1097/CCM.0b013e31827e83af

35. Acute Respiratory Distress Syndrome Network. Ventilation with lower tidal volumes as compared with traditional tidal volumes for acute lung injury and the acute respiratory distress syndrome. N Engl I Med 2000;342(18):1301-1308. https://doi.org/10.1056/NEJM200005043421801 